\title{
A resolução de problemas de geometria com o auxílio da plataforma Virtual Math Teams e do software GeoGebra
}

\author{
Bruna Luana Züge (D) Renan Severo Ferreira (D) \\ Carmen Vieira Mathias
}

Sabrina Paris de Lima(iD

\section{Resumo}

No ano de 2020, devido à pandemia (Covid-19), a maneira como se ensina e aprende teve que ser repensada nos diferentes níveis de ensino. Um dos caminhos foi a utilização das tecnologias, por meio de aulas síncronas e assíncronas. Nesse sentido, este trabalho é um relato de uma atividade desenvolvida na disciplina de Geometria Plana e Espacial de um Programa de Pós-Graduação. Com o auxílio do software GeoGebra e por meio da plataforma Virtual Math Teams (VMT) foi realizada a releitura de um artigo envolvendo problemas geométricos. A utilização da VMT mostrou-se adequada para a realização de um trabalho online e em grupo, e o software GeoGebra foi importante aliado para facilitar a compreensão da resolução dos problemas. Neste sentido, considera-se que ações como essas podem ser uma opção para o ensino remoto, pois colaboram com a visualização, assimilação da resolução de problemas e o entendimento dos alunos.

Palavras-chave: Tecnologias; Geometria; Visualização; GeoGebra.

\begin{abstract}
In the year 2020, due to pandemic (Covid-19) the way you teach and learn had to be rethought in the different levels of education. One of the ways was the use of technologies, through synchronous and asynchronous classes. In this sense, this work is an account of an activity developed in the discipline of Flat and Spatial Geometry of a Graduate Program. With the help of GeoGebra software and through the Virtual Math Teams platform (VMT) was performed the reading of an article involving geometric problems. The use of VMT proved to be suitable for carrying out online and group work, and the GeoGebra software was an important ally to facilitate the understanding of problem solving. In this sense, it is considered that actions like these can be an option for remote teaching, as they collaborate with visualization, assimilation of problem solving and students' understanding.
\end{abstract}

Keywords: Technologies; Geometry; Visualization; GeoGebra. 


\section{Introdução}

Atentos às constantes mudanças e avanços em Tecnologias Digitais (TD), pesquisadores e professores de matemática sentem-se obrigados a acompanhar esse processo, utilizando tais recursos a seu favor.

É senso comum que a integração da tecnologia na educação matemática pode ser realizada por meio do uso de computadores e/ou dispositivos móveis nos ambientes de ensino e aprendizagem, de forma presencial ou virtual. Inclusive, estudos apontam as vantagens do uso de softwares em sala de aula para auxiliar no processo de ensinar e aprender matemática. Por exemplo, [5] relatam que o uso do computador é mais eficaz do que a abordagem tradicional de aprendizagem, em especial, em transformações geométricas, polígonos, prismas e pirâmides.

Levando-se em consideração que a utilização de softwares é um recurso que facilita a visualização, como conclui [12] trata-se de um componente efetivo na compreensão dos conceitos geométricos. Dessa maneira, [6] ) afirma que os softwares de geometria dinâmica oferecem um ambiente didático eficaz devido à sua capacidade de interagir com materiais digitais e permitir que objetos geométricos possam ser vistos dinamicamente. Justifica-se, assim, a escolha em trabalhar com o software GeoGebra na presente pesquisa.

Tendo em vista as considerações precedentes, uma finalidade do presente artigo é apresentar a releitura de alguns problemas propostos em [13], bem como relatar a realização dessa experiência durante período de ensino remoto. O artigo em questão faz parte de uma coletânea publicada em 1987, no National Council of Teachers of Mathematics (Conselho Nacional de Professores de Matemática) dos Estados Unidos, a qual apresenta 20 trabalhos de diferentes pesquisadores da área de Educação Matemática. Em especial, [13], cujos problemas foram escolhidos para releitura, discorre a respeito da resolução de problemas e aplicações envolvendo atividades de geometria, procurando estimular a flexibilidade e o raciocínio dos alunos.

Outro ponto a ser destacado é com relação ao atual momento vivenciado, onde não é possível realizar encontros para concretizar trabalhos e tarefas, fazendo com que alunos e professores busquem novas ferramentas para o ensino e a aprendizagem. Para este trabalho, foi utilizado o Virtual Math Teams (VMT), um projeto desenvolvido com a colaboração de pesquisadores da Drexel University, do Math Forum e de algumas universidades brasileiras. Trata-se de uma plataforma gratuita e, para utilizá-la, deve-se fazer um cadastro simples. No VMT é possível utilizar os softwares GeoGebra e/ou Desmos como espaços de trabalho, tendo como objetivo estimular de forma colaborativa a discussão de problemas matemáticos em um ambiente virtual. Nessa plataforma, é permitido criar cursos, salas e atividades cooperativas. Ou seja, trata-se de um ambiente em que todos os participantes possuem acesso, de forma que os professores possam visualizar as alterações realizadas por cada participante.

Nos próximos tópicos, é apresentada uma breve revisão bibliográfica sobre Tecnologias Digitais e Visualização, teoria esta que traz um embasamento para a presente pesquisa. Além disso, expõe-se a metodologia do estudo e, por fim, a descrição da resolução de quatro problemas sugeridos no artigo com o uso do software GeoGebra.

\section{Tecnologias Digitais e Visualização}

Nos tempos atuais, vive-se em uma sociedade cada vez mais tecnológica, na qual as inovações, além de estarem presentes em nossas ações do dia a dia, também acabam por adentrar ao âmbito do 
ensino e da aprendizagem, em diversas áreas do conhecimento. Segundo Kenski (2013), Conforme $[7]$

O crescimento acelerado das inovações nas tecnologias digitais nos últimos anos ampliou o número de ações educacionais via internet. As facilidades de interação e comunicação oferecidas pela web redefiniram estratégias e procedimentos para o oferecimento de processos de capacitação dinâmicos, de acordo com as necessidades e os interesses dos participantes. (Kenski, 2013, p. 60)

Todas essas novas facilidades e diversidades de interações acabam por despertar curiosidade em diversos profissionais. Contudo, a inclusão dos novos recursos necessita de uma infraestrutura adequada no espaço escolar, assim como de preparação e de responsabilidade no momento de seu uso. Tendo em vista que essas tecnologias implicam a criação de outros espaços, é interessante, ainda, que existam metodologias diferenciadas daquelas que se empregam no ensino puramente presencial, para a realização de ações como as desenvolvidas no presente artigo.

Nessa pesquisa, o artigo escolhido para releitura ([13]) apresenta problemas de diferentes tópicos em geometria, os quais procuram estimular o raciocínio dos alunos, permitindo, para a sua resolução, diferentes estratégias. Com relação a isso, [8] enfatiza que:

\section{Desse modo o ensino da Geometria deve proporcionar ao educando momentos que o levem a desenvolver habilidades espaciais e estimulá-lo a compreender, descrever $e$ representar o espaço que o cerca, observando semelhanças, diferenças e regularidades, além de construir outras relações pertinentes. (Oliveira e Leivas, 2017, p. 109)}

Considerando-se, a partir dessa colocação, que com a utilização de TD no processo de ensino de geometria será possível alcançar, de uma maneira mais satisfatória, o aprendizado por parte dos alunos, é possível realizar uma relação com o seguinte fragmento:

Os softwares de Geometria Dinâmica proporcionam novas possibilidades de exploração e experimentação de estratégias didáticas a partir do movimento produzido em construções geométricas. Essa estrutura dinâmica proporciona a visualização das construções, de seus elementos e propriedades, tanto no espaço bidimensional quanto no tridimensional. Os softwares gráficos para ensino e aprendizagem de Matemática possibilitam a visualização das distintas representações de um objeto simultaneamente $e$, por conseguinte, a transição entre as múltiplas representações. (Pinto; Mota; Ferreira, 2019, p. 192)

Sendo assim, com o auxílio de softwares, tem-se uma melhor visualização dos processos envolvidos durante a execução de atividades envolvendo geometria, e também outros conteúdos matemáticos.

Além do mais, o software de matemática dinâmica GeoGebra, pelo qual se optou utilizar neste trabalho, é gratuito e fornece janelas de visualização tanto gráfica quanto algébrica. Além disso, permite a visualização de elementos bidimensionais e tridimensionais. 


\section{Procedimentos Metodológicos}

Este artigo surgiu por meio de um trabalho proposto na disciplina de "Geometria Plana e Espacial", de um programa de Pós-Graduação em Educação Matemática de uma universidade pública, no primeiro semestre de 2020. O objetivo do trabalho era apresentar uma releitura de um artigo embasado em tecnologias, história da matemática e metodologia de resolução de problemas. Para esse fim, escolheu-se o sétimo artigo da coletânea "Aprendendo e Ensinando Geometria" [11] intitulado "Problemas de Geometria criativos podem levar à resolução criativa de problemas criativos". O estudo discorre sobre a resolução de problemas e aplicações envolvendo atividades de Geometria, procurando, assim, estimular a flexibilidade e o raciocínio dos alunos.

É interessante ressaltar que as aulas no referido semestre foram realizadas de forma remota, via ambiente Moodle. Nesse período, os autores do grupo ficaram responsáveis por uma releitura dos problemas descritos no artigo, de uma maneira tecnológica, de forma que esta pudesse lhes dar uma maior dinamicidade e possibilidade de movimentação. Desse modo, buscou-se, por meio dessa adaptação, trazer uma maior facilidade na compreensão e no entendimento do que se pede em cada um dos questionamentos. Nesse sentido, acreditou-se que a tecnologia que poderia auxiliar nesse processo seria o software de matemática dinâmica GeoGebra.

Além disso, devido à situação vivenciada (epidemia do Covid-19), onde encontros presenciais estavam fora de cogitação, optou-se pela utilização do Virtual Math Teams (VMT) para a realização das construções geométricas, de modo que essa plataforma possibilitou um ambiente colaborativo, onde applets do GeoGebra puderam ser alterados em tempo real por todos os integrantes do trabalho.

A escolha dos problemas que seriam construídos, descritos no artigo citado, foi realizada de acordo com o interesse de cada um dos membros do grupo. Em seguida, tem-se realizada uma descrição detalhada de cada problema, conforme se apresenta na seção a seguir. Além da explicação e do debate a respeito da resolução do problema, são explorados os processos de construções envolvidos e seus desdobramentos decorrentes.

\section{Desenvolvimento}

Nesta seção, apresenta-se a resolução de quatro problemas propostos no tópico "Coletânea de Problemas", constantes em [13]. Observa-se que a escolha dos problemas foi aleatória e que para resolvê-los foi utilizado o software GeoGebra com o auxílio da plataforma VMT. Tal escolha dos problemas foi aleatória, e, para resolvê-los, foi utilizado o software GeoGebra, com o auxílio da plataforma VMT. Assim, procurou-se trazer uma noção de como a resolução de problemas sobre o conteúdo de geometria pode ser dinâmica e de fácil visualização com a utilização de softwares. O primeiro problema escolhido é um clássico, que frequentemente aparece em concursos ou livros didáticos do ensino médio.

Problema 1. Dois quadrados congruentes de $6 \mathrm{~cm}$ x $6 \mathrm{~cm}$ sobrepõem-se, conforme mostra a figura. Um vértice de um dos quadrados está no centro do outro quadrado. Qual é o maior valor possível da área hachurada? (O primeiro quadrado é móvel, mantendo-se fixo apenas o vértice que está no centro do outro, conforme ilustra a figura 1.) 


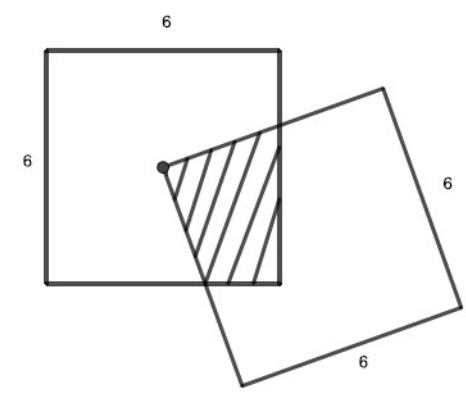

Figura 1: Quadrados sobrepostos de lados $6 \mathrm{~cm}$.

Salienta-se que os problemas não possuem resolução, sendo essa elaborada pelos autores, que eram membros do grupo. Para resolver tal problema, inicialmente, realizou-se a construção dos quadrados iniciais sobrepostos. Além disso, foi usada a ferramenta Controle deslizante, na função ângulo ( $\alpha$ variando de $0^{\circ}$ a $360^{\circ}$ ), para efetuar a rotação do quadrado polígono EFIJ) que possui um de seus vértices no centro do outro quadrado (polígono ABCD). Para realizar a rotação, na caixa de entrada digitou-se o comando Girar ( $\langle$ Objeto $\rangle,\langle\hat{A}$ ngulo $\rangle,\langle$ Eixo de Rotação $\rangle$ ), como ilustra a Figura 2.

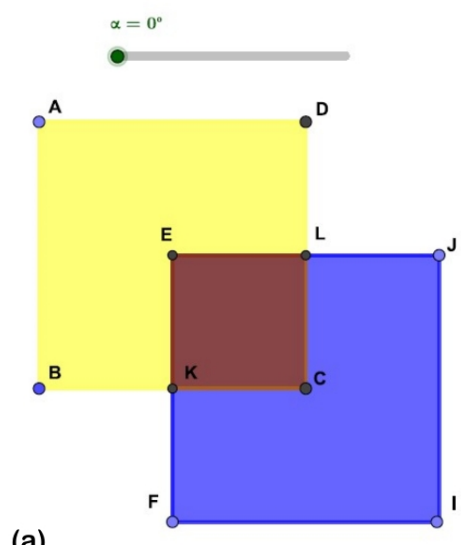

(a)

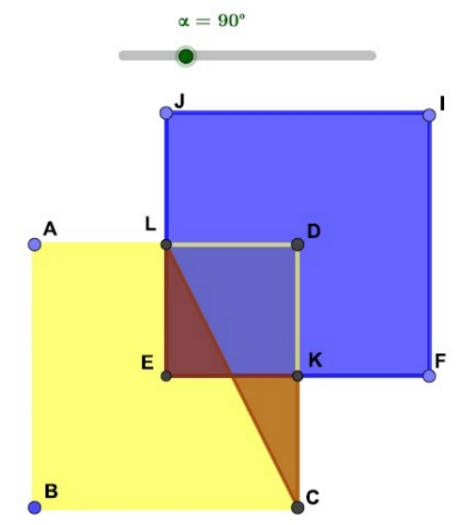

(b)

Figura 2: Polígono EKCL resultante da interseção entre os polígonos ABCD e EFIJ (a) e Polígono EKCL não convexo resultante da rotação anti-horária do polígono EFIJ (b).

Além disso, como o objetivo era determinar o maior valor da área hachurada, ou seja, a área da interseção dos dois quadrados, foi necessário destacar essa área. Sendo assim, inicialmente foram marcadas as interseções K e L dos quadrados. Depois disso, foi criado um polígono, o qual era determinado pelos centros e as interseções dos dois quadrados, de modo a resultar na área de interesse (Figura 2(a)). 
Porém, ao rotacionar o quadrado, pode-se observar que foram obtidos polígonos não convexos, devido à maneira como foi definida a ordem dos vértices, como ilustra a Figura 2(b). Desta maneira, foi necessário realizar alterações nas propriedades no polígono criado, mais especificamente na aba "Condições para Exibir Objeto(s)". Sendo assim definiu-se que o polígono LCKE deveria aparecer em intervalos específicos do ângulo $\alpha$ (ângulo de rotação). O intervalo definido foi de $0^{\circ} \leq \alpha \leq 44^{\circ} \vee 315^{\circ} \leq \alpha \leq 360^{\circ}$ pois, posteriormente a formação do triângulo, quando $\alpha=44^{\circ}$ (Figura 3), o polígono passa a ser não convexo. Dessa forma, para valores maiores do que $44^{\circ}$ foi definido um novo polígono.

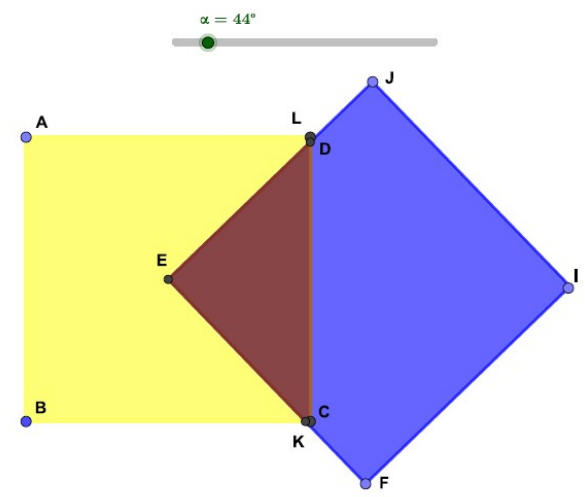

Figura 3: Triângulo que é obtido quando $\alpha=44^{\circ}$.

Observa-se a inclusão do símbolo $\vee(\mathrm{ou})$ no comando apresentado anteriormente. TAl inserção deve-se ao fato de que, quando $\alpha=315^{\circ}$, ocorre a formação de um novo triângulo, de forma que se utilizou o mesmo polígono, retornando à posição inicial.

Após, foram traçadas retas em cada um dos polígonos convexos (definidos dentro dos respectivos intervalos que aparecem), entre o ponto E e os pontos L e K, definidos anteriormente (Figura 4(a). Sobre essas retas foram construídos segmentos com as novas interseções formadas. Depois, as retas que serviram de suporte foram ocultadas (Figura 4(b). A partir dessas novas subdivisões, fica claro que o quadrado é dividido em 4 partes iguais. 
(a)

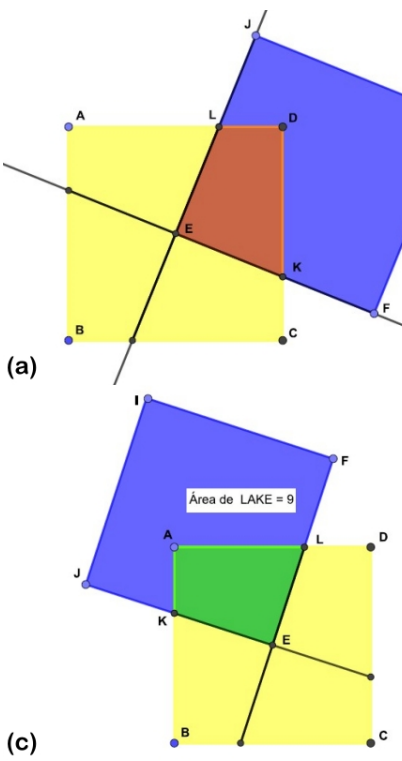

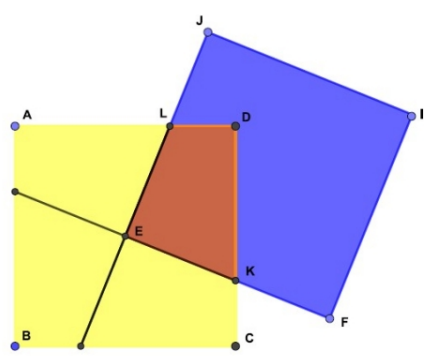

(b)

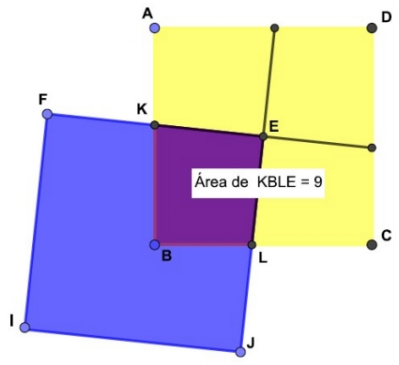

Figura 4: Retas e segmentos definidos sobre as retas (a) e (b) e área da parte hachurada (c) e (d).

Observa-se que, além de facilitar o processo de resolução do problema, a construção descrita facilita o processo de visualização dos elementos que o compõem. Desse modo, como a área total do quadrado é 36 unidades de área, e cada parte hachurada ocupa apenas $\frac{1}{4}$ da área total, então a área em destaque será 9 unidades de área independentemente da sua forma, como ilustram as figuras $4(\mathrm{c})$ e $4(\mathrm{~d})$.

O segundo problema escolhido também versa sobre o tema área, e possui o seguinte enunciado:

Problema 2. Dado um quadrado unitário, ache a área do quadrado interior S, que é determinado unindo-se cada vértice do quadrado original ao ponto médio do lado não adjacente (sentido horário)

A Figura 5 ilustra a situação descrita.

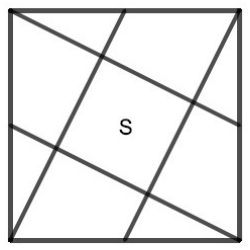

Figura 5: Quadrado unitário. 
Assim, no GeoGebra, visando a ilustração do problema, foi construído um controle deslizante $\alpha$ variando de $180^{\circ}$ a $360^{\circ}$. Em seguida utilizando ferramenta polígono foram criados quatro triângulos conforme ilustra a figura 6(a) e foi utilizado o comando Girar (<Objeto $\rangle,<\hat{A n g u l o}\rangle$, $<$ Eixo de Rotação $>$ ), para rotacionar os triângulos em $180^{\circ}$, como ilustram as figuras 6(b) e 6(c).

(a)

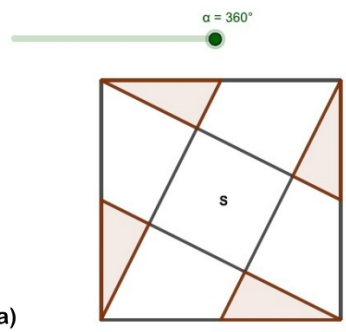

(c)

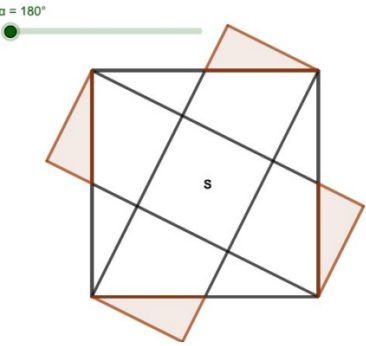

(b)

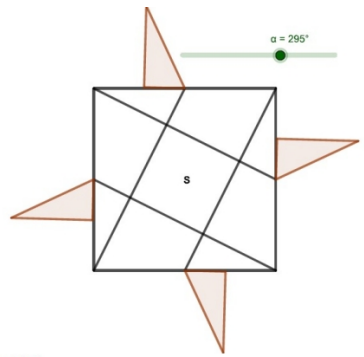

(d)

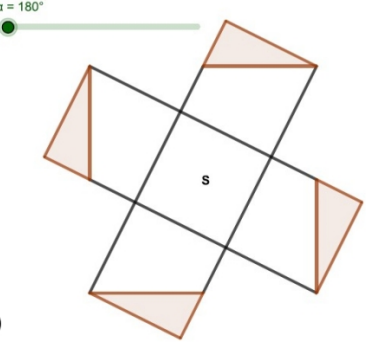

Figura 6: Triângulos construídos (a), rotação dos triângulos (b) e (c) e quadrados congruentes (d).

Observa-se (Figura 6(d) que, a partir da rotação dos triângulos, foram formados cinco quadrados congruentes. Com isso, percebe-se que a área do quadrado maior é cinco vezes a área procurada. Logo, a área de S é um quinto da área do quadrado.

Essa construção foi realizada de forma colaborativa, utilizando-se a plataforma VMT, fundamental para que os membros do grupo discutissem as estratégias utilizadas na resolução do problema.

O terceiro problema escolhido trata do tema geometria espacial, mais especificamente sobre a distância entre dois pontos. Em particular, essa questão é importante no contexto do trabalho, pois os processos de ensino e de aprendizagem da visualização espacial são objeto de atenção em muitas pesquisas ([2]; [3] e [10]), de importância formação na professores de matemática. O referido problema possui o seguinte enunciado

Problema 3. Cada aresta do cubo da ilustração (Figura 7) mede $6 \mathrm{~cm}$. Ache a distância do ponto médio de uma das diagonais das faces ao vértice mais distante do cubo. 


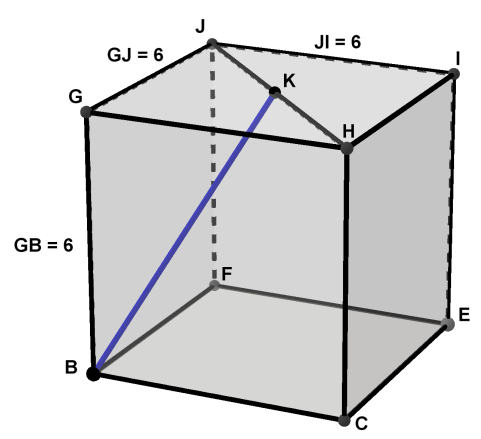

Figura 7: Cubo cujas arestas medem $6 \mathrm{~cm}$ e segmento BK definido.

Para iniciar a resolução deste problema, primeiramente realizou-se a construção de um cubo de aresta 6, a diagonal da face superior e o ponto médio deste segmento. O fato inicial que foi preciso atentar é que o valor da diagonal da face é conhecido (correspondente a $6 \sqrt{2}$ unidades de medidas). Contudo, esse valor será coincidente em todas as diagonais das faces quadradas do poliedro, de modo que se destacaram as diagonais JB e HB, como ilustra a Figura 8.

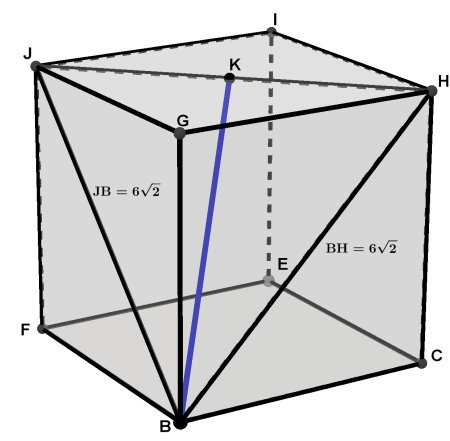

Figura 8: Diagonais das faces.

Observa-se que por meio da união das três diagonais (JB, HB e JH) forma-se um triângulo equilátero JBH de lado $6 \sqrt{2}$, de modo que o segmento BK (procurado) é a mediana do segmento JH. Como, porém, o triângulo obtido é equilátero, segue que as alturas, medianas e bissetrizes coincidem. Sendo assim, pode-se considerar que BK é a altura do triângulo JBH, ou seja, pode ser determinado como sendo:

$$
\mathrm{BK}=\frac{6 \sqrt{6}}{2}=3 \sqrt{6}
$$

Ao se pensar que nem sempre os alunos conseguem visualizar os elementos de uma construção 
espacial, ou como um professor no ensino médio poderia apresentar esse problema aos alunos, optou-se por realizar, na mesma construção, a planificação do cubo, como ilustra Figura 9(b).

(a)

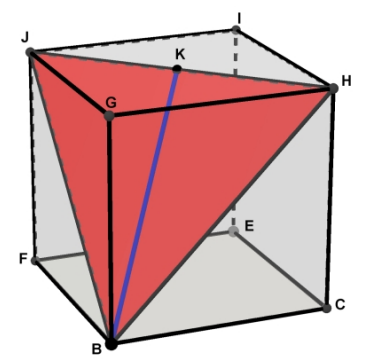

(b)

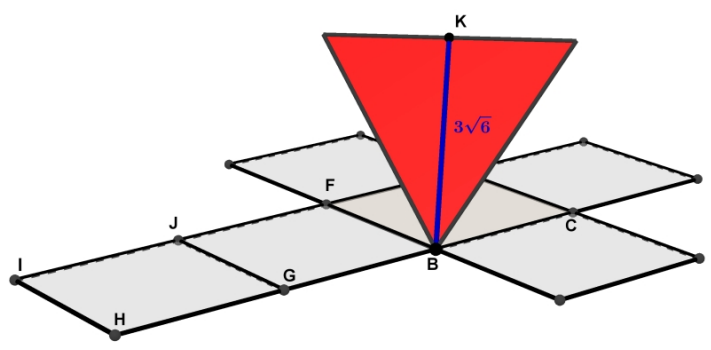

Figura 9: Triângulo equilátero formado pelas diagonais e altura desejada (a) e planificação do cubo para evidenciação da construção interna (b).

Observa-se que, no caso da resolução desse problema usando o VMT, como os principais conhecimentos envolvidos na resolução eram conhecidos, ao planificar o cubo, o grupo foi capaz de variar os aspectos elencados no problema, gerando uma nova abordagem visual, o que foi crucial no entendimento da questão por parte de um dos membros do grupo. Esse tipo de interação só foi possível graças ao dinamismo do software GeoGebra e a colaboração no VMT. Outro aspecto considerado pelo grupo foi a possibilidade de construir um cubo com o lado variável (a partir de um controle deslizante), o que permitiria uma variação nos parâmetros e uma generalização do resultado obtido. O último problema escolhido pode ser facilmente resolvido usando conhecimentos de geometria analítica. Devido,porém, devido à familiaridade e gosto pessoal de um dos autores por construções geométricas, a resolução apresentada utiliza esses processos. O problema possui o seguinte enunciado:

Problema 4. Dado o triângulo $\mathrm{ABC}$ de vértices $\mathrm{A}(-3,-10), \mathrm{B}(-12,2)$ e $\mathrm{C}(12,10)$, ache o ponto em que a bissetriz do ângulo $\widehat{\mathrm{A}}$ intercepta $\mathrm{BC}$.

Para resolver o problema, foi construído um triângulo ABC com as coordenadas propostas (Figura 10), e também a bissetriz do ângulo $\widehat{A}$. 


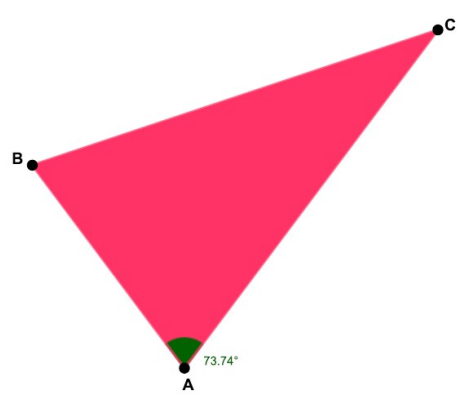

Figura 10: Triângulo ABC.

Para a construção da bissetriz, em vez de utilizar a ferramenta disponível no software, optouse por realizar a construção geométrica. Para isso, em um primeiro momento, marca-se uma circunferência de centro em A (Figura 11(a)) e a medida do raio arbitrária ${ }^{1}$, interceptando o triângulo nos pontos $\mathrm{D}$ e E referentes aos lados $\mathrm{AB}$ e $\mathrm{AC}$ do triângulo. Feito isso, constroem-se mais duas circunferências, as quais terão como centro os pontos D e E . Nesses, tem-se como medida do raio a distância do centro da circunferência até o ponto da interseção de um dos lados do triângulo com a primeira circunferência, como ilustram as Figuras 11(b) e 11(c).

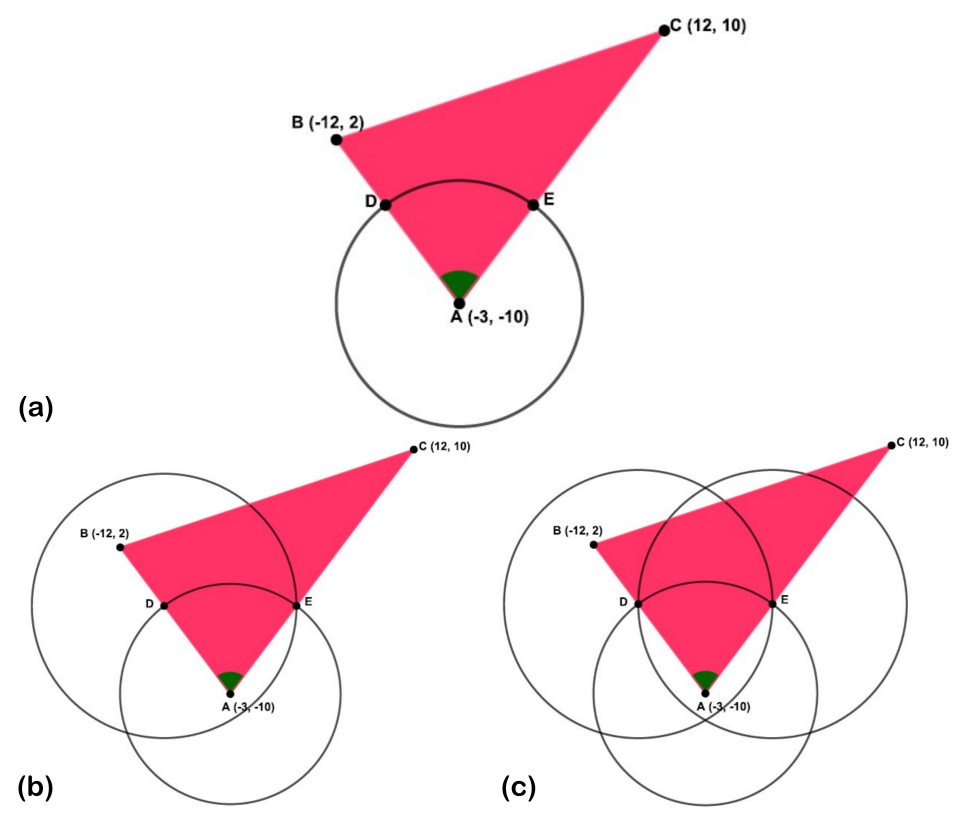

Figura 11: Construção da bissetriz.

\footnotetext{
${ }^{1}$ Mas, que não ultrapasse as medidas dos lados $\mathrm{AB}$ e $\mathrm{AC}$, pois precisamos das interseções resultantes, caso contrário seria necessário realizar prolongamentos desses segmentos.
} 
O próximo passo foi a construção da semirreta que divide o ângulo $\widehat{A}$ em duas partes iguais. Observou-se que a semirreta tem origem no ponto A e passa pelo ponto de interseção das últimas duas circunferências construídas. A interseção dessa semirreta com o lado BC é o ponto procurado (ponto $\mathrm{G}$ de coordenadas $(-3,5)$ ) como ilustra a Figura 12.

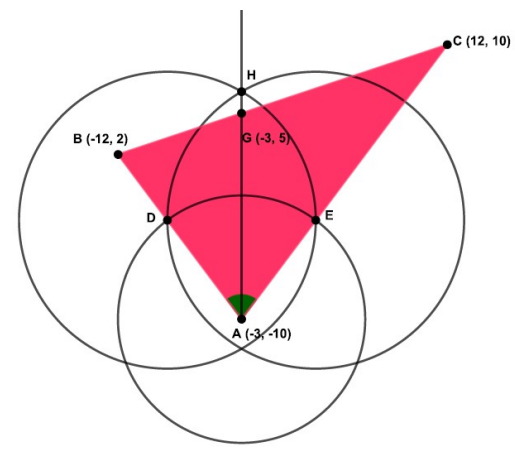

Figura 12: Construção da bissetriz.

Essa construção é relativamente simples e conhecida, mas acredita-se importante retomá-la, pois, durante a disciplina cursada, houve um momento em que foi necessário recordá-la, visto que nem todos os alunos (que são professores de matemática) tinham conhecimento sobre ela.

\section{Conclusões}

Neste artigo, apresentou-se uma releitura de alguns problemas propostos no livro Aprendendo e Ensinando Geometria, de modo que procurou-se realizar a resolução destes utilizando o software de matemática dinâmica GeoGebra, assim como a plataforma VMT. Além do livro citado durante o trabalho sugere-se àqueles em construções geométricas outras referências, como [4], uma vez que nele é possível estudar as construções, de uma maneira clássica. Outro material interessante e com uma abordagem mais moderna é [1], que propõe auxiliar o professor no processo operacional do software GeoGebra, ao mesmo tempo em que apresenta atividades que exploram construções, manipulações e visualizações de diversos entes geométricos, proporcionando um passo além no processo de produção de conhecimento dos estudantes.

Considerando o atual momento de pandemia, no qual todas as atividades presenciais foram suspensas, o VMT foi um aliado importante para a discussão das questões e a constituição das construções em grupo no GeoGebra. Com relação à resolução dos problemas, notou-se que, por meio do uso do software, as construções iniciais deram um aporte visual muito mais significativo para a compreensão do que estava sendo proposto. Nesse sentido, considera-se que esta ação poderia ser vista como uma opção para o ensino remoto, pois colabora com a visualização e a assimilação da resolução de problemas, assim como ajuda os alunos no desenvolvimento dessas habilidades, aprimorando seu entendimento.

A realização dessas construções em grupo, por meio do software GeoGebra e do VMT, mostrou que estratégias didáticas como a apresentada no presente artigo podem ser utilizadas em todos os níveis de ensino, a fim de facilitar a comunicação e a colaboração entre pares. Sendo assim, defende-se que ações nessa direção possuem um grande valor nos processos de ensinar e de aprender 
matemática, em particular geometria. Portanto, são necessárias em uma situação como a que a humanidade está enfrentando.

\section{Referências}

[1] Araújo, L.C.L. de; Nóbrega, J. C. Aprendendo Matemática com o GeoGebra. São Paulo: Editora Exato, 2010.

[2] Arrieta, M. "La capacidad espacial en la educación matemática: estructura y medida". Educación Matemática, vol. 1, no 18 , pp. 99-132, 2006.

[3] Battista, M. T. "The Development of Geometric and Spatial Thinking. en F. Lester (ed.)". Second Handbook of Research on Mathematics Teaching and Learning, Charlotte, NC, Information Age Publishing, pp. 843-908, 2007.

[4] Braga, T. Desenho Geométrico Linear: problemas de desenho linear geométrico.14. ed. São Paulo: Icone, 1997.

[5] Dogan, M.; İcel, R. "The role of dynamic geometry software in the process of learning: GeoGebra example about triangles". Journal of Human Sciences, v. 8, nํ․ 1, pp. 1441-1458, 2011.

[6] Ibili, E. "The use of dynamic geometry software from a pedagogical perspective: current status and future prospects". Journal of Computer and Education Research, v. 7, no․ 14, pp. 337-355, 2019.

[7] Kenski, V. M. Tecnologias e tempo docente. Campinas, SP: Papirus, 2013.

[8] Oliveira, M. T.; Leivas J. C. "Visualização e Representação Geométrica com suporte na Teoria de Van Hiele". Ciência e Natura, v. 39, nํ 1, pp. 108-117, 2017.

[9] Pinto, R. L.; Mota, J. F.; Ferreira, R. D. "Visualização E Pensamento Geométrico Na Geometria Em Movimento". Revista de Ensino de Ciências e Matemática, v. 10, no 2, pp. 188-203, 2019.

[10] Presmeg, N. C. Research on visualization in learning and teaching mathematics: emergence from psychology. In A. Gutiérrez y P. Boero (eds.) andbook of research on the psychology of mathematics education, Dordrecht, Sense Publishers, pp. 205-235, 2006.

[11] Lindquist, M. M.; Shulte, A. P. Aprendendo e ensinando geometria. São Paulo: Atual, 1994.

[12] Lopes, M. M. "Sequência didática para o ensino de trigonometria usando o software GeoGebra". "Bolema: Boletim de Educação Matemática", v. 27, nº 46, pp. 631-644, 2013.

[13] Milauskas, G. A."Problemas de geometria criativos podem levar à resolução criativa de problemas". In Lindquist, M. M.; Shulte, A. P. Aprendendo e ensinando geometria. São Paulo: Atual, 1994. 
Bruna Luana Züge Universidade Federal de Santa Maria $<$ brunazuge28@gmail.com>

Renan Severo Ferreira Secretária de Educação do Estado do Rio Grande do Sul $<$ renansevero@hotmail.com>

Sabrina Paris de Lima Universidade Federal de Santa Maria <sabrinaparisdelima@outlook.com>

Carmen Vieira Mathias Universidade Federal de Santa Maria <carmen@ufsm.br>

Recebido: $15 / 10 / 2020$

Publicado: $02 / 02 / 2021$

Chamada Temática "Experiências didáticas em Matemática no período de isolamento social" 